\title{
Technique and Computational Improvements in 4D STEM and Cross-Correlation Analysis
}

Tristan O'Neill ${ }^{1}$, B. C. Regan ${ }^{2}$ and Matthew Mecklenburg ${ }^{3}$

${ }^{1}$ University of California, Los Angeles, Los Angeles, California, United States, ${ }^{2}$ Department of Physics and Astronomy, University of California, Los Angeles, California 90095, United States, United States, ${ }^{3}$ The Aerospace Corporation, United States

Lattice parameter measurements can be used to map temperature [1] and strain [2] in crystals. Even in single crystals, lattice parameters have been observed to vary at the parts-per-thousand level. One can determine the lattice parameters of a single crystal with convergent-beam electron diffraction (CBED), where one scans a nanometer-sized probe across the sample and collects a diffraction pattern at each point. But a compromise must often be made: decreasing the probe convergence angle improves the disk identification but hurts resolution. However, no compromise is necessary in silicon. With a beam along the [100] zone axis, the diffraction disks do not overlap with a convergence angle large enough to achieve lattice resolution ( $\alpha=10$ mrads) (Fig. 1). We can determine the lattice parameter with subpixel resolution via cross-correlation and Gaussian fitting with a precision of less than 0.2 parts per thousand. Additionally, under these conditions a separate reference image is not required for the cross-correlation.

With normal CPU-based computational methods, 4DSTEM cross-correlation analysis can take a prohibitively long time. Leveraging GPUs to perform the analysis can speed up the processing by orders of magnitude, even with GPUs that are five years outdated, and even with the new bottleneck being the data transfer time between the RAM and GPU. In our tests, an outdated GPU far outperformed a recently released CPU. The newest generation of GPUs would likely be able to nearly eliminate this bottleneck. Here we show preliminary results of analysis done in Python using the open-source module CuPy for GPU programming. We compare the performance of the standard NumPy module.

Diffraction data was acquired on a $\mathrm{KOH}$-etched piece of silicon wafer at a thin, electron-transparent edge. The diffraction disks were filtered in reciprocal space (Fig. 1a), using a Hanning window followed by a bandpass filter (Fig. 1b). A reference image was created by taking the mean across every diffraction image in the set, masking it to retain only the central disk of the mean, and applying the same filters as above (Figs. 1c-d). Cross-correlation analysis with the reference image was performed on each diffraction image to determine the spacing between diffraction pattern disks (Fig. 2). Because the dataset is large, GPUs were leveraged to perform the cross-correlation analysis more efficiently. The hope is that crosscorrelation analysis will yield local lattice parameters, and that from these it will be possible to determine the temperature and the strain of the sample without compromising lattice resolution. 

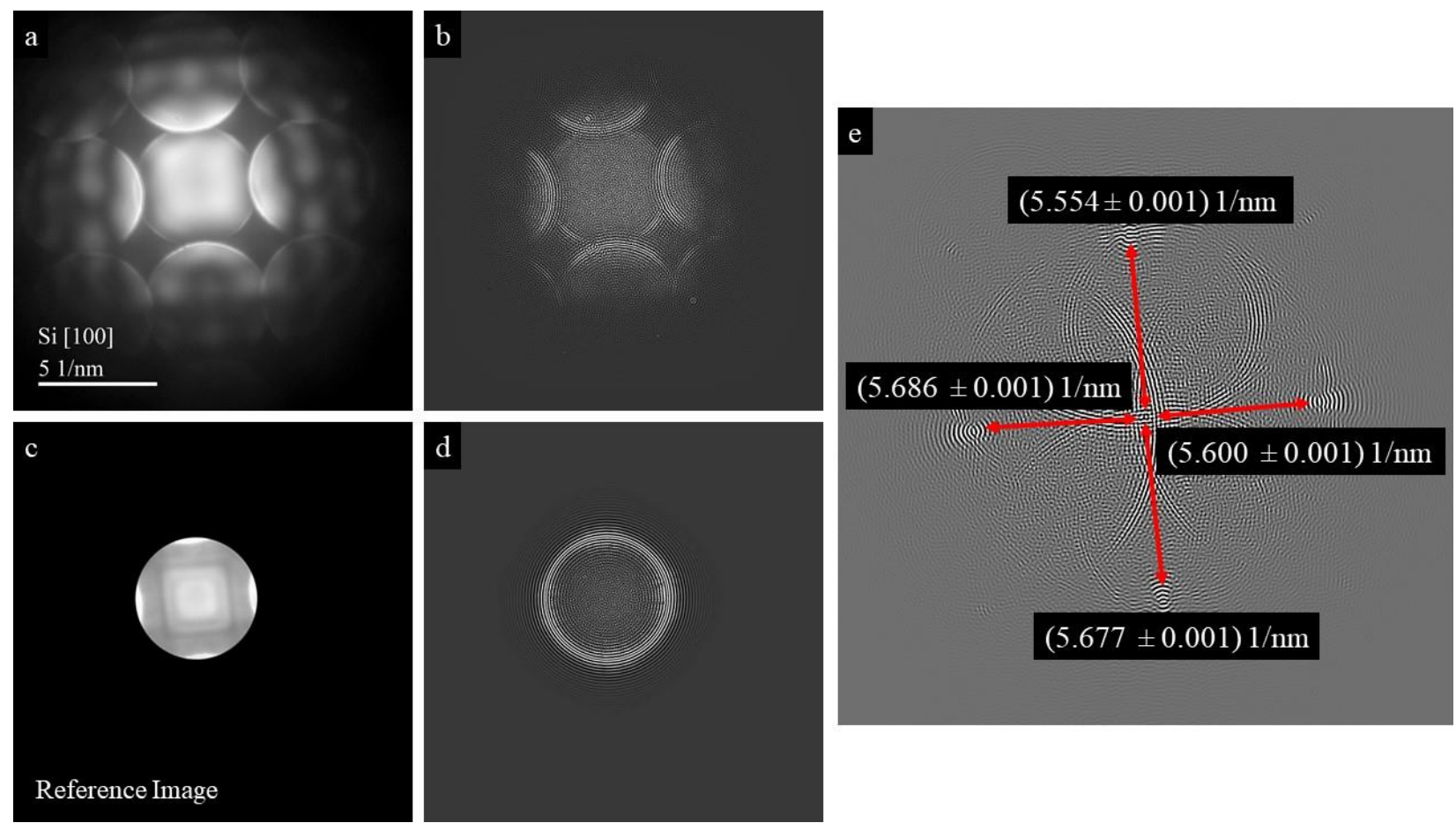

Figure 1. Example diffraction pattern from a 4D STEM dataset. a: An example CBED pattern taken from a Si crystal along the [100] zone axis. b: The example pattern a, after application of a Hanning window and a bandpass filter in preparation for the cross-correlation analysis. c: The reference pattern for the cross correlation. This image was obtained by averaging all of the CBED patterns obtained from this crystal and applying a mask to retain only the central disk. d: The reference pattern c, after filtering with a Hanning window and bandpass filter in preparation for cross correlation analysis. e: The resultant cross correlation. Distances between the diffraction disks and the central disk (as found by Gaussian fitting) are indicated.

\section{References}

[1] F. Gaulandris et al., Microscopy and Microanalysis, 26(1) (2020), p. 3-17.

[2] C. Ophus, Microscopy and Microanalysis, 25(3) (2019), p. 563-582.

[3] I. Thomas and M. Schmid, Microscopy and Microanalysis, 1(4) (1995), p. 167-173

[4] The data was acquired at the Core Center of Excellence in Nano Imaging (CNI), University of Southern California. This work was supported by National Science Foundation (NSF) Science and Technology Center (STC) award DMR-1548924 (STROBE) and by NSF award DMR-2004897. 